\title{
Depression negatively affects patient- reported knee functional outcome after intraarticular hyaluronic acid injection among geriatric patients with knee osteoarthritis
}

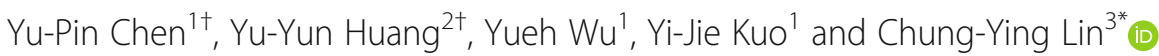

\begin{abstract}
Purpose: Intraarticular hyaluronic acid injection (IAHA) is a popular treatment for knee osteoarthritis (OA). This study investigates whether depression, anxiety, and pain affect self-reported knee function in geriatric OA people who have received IAHA.

Methods: Through convenience sampling, 102 geriatric patients (mean age $=70.91 \pm 7.19 ; 28$ males) with knee OA who had undergone IAHA participated in this study. All participants self-reported depression using the Geriatric Depression Scale (GDS), anxiety using the State-Trait Anxiety Inventory (STAl), knee function using the Western Ontario and McMaster University Osteoarthritis Index (WOMAC) and the International Knee Documentation Committee subjective knee evaluation form (IKDC), and pain severity using the Visual Analogue Scale (VAS). They completed the aforementioned questionnaires at baseline before injection and then again at 2-, 4-, and 6-month follow-ups.
\end{abstract}

Results: Depression was significantly associated with IKDC, WOMAC physical function subscale, and total WOMAC scores. Anxiety was only significantly associated with the WOMAC pain subscale score. Pain severity was significantly associated with IKDC, WOMAC stiffness subscale, WOMAC physical function subscale, and total WOMAC scores.

Conclusion: Given that depression negatively affected patient-reported knee function among geriatric OA patients who had undergone IAHA, further attention should be paid to the depressive status of this population.

Keywords: Hyaluronic acid, Anxiety, Depression, Knee osteoarthritis, Pain

\section{Background}

Osteoarthritis (OA) commonly affects middle-age to elderly people, with an incidence of symptomatic knee OA up to $10 \%$ of men and $13 \%$ of women aged 60 years or older [1-3]. Although the end stages of progressive knee $\mathrm{OA}$ are generally managed by joint replacement, there are still various conservative treatments before end stage

\footnotetext{
* Correspondence: cylin36933@gmail.com

${ }^{\dagger}$ Yu-Pin Chen and Yu-Yun Huang contributed equally to this work.

${ }^{3}$ Department of Rehabilitation Sciences, Faculty of Health and Social

Sciences, The Hong Kong Polytechnic University, 11 Yuk Choi Rd, Hung Hom, Hong Kong

Full list of author information is available at the end of the article
}

of knee OA with diverse short-term effects [4]. Thus, healthcare providers should understand and evaluate all the possible advantages and disadvantages among different treatments to make the optimal decision-making. One common conservative treatment, intraarticular hyaluronic acid injection (IAHA), improves joint lubrication, increases synovial fluid viscosity, and reduces pain by its analgesic and anti-inflammatory effects $[5,6]$. However, controversy exists on the efficacy of IAHA especially in the geriatric population, who are vulnerable to chronic pain as a result of knee OA [7]. In fact, younger patients with less severe structural damage are 
reported to gain the best benefit from IAHA treatment than the elderly population [8]. To maximize the treatment effects of IAHA, factors that potentially hinder those treatment effects for the elderly must be investigated. Specifically, we proposed to investigate the roles of psychological health among these patients as the effects of psychological health on knee functions are understudied in the patients with knee OA.

Moreover, knee OA is reportedly associated with depressive symptoms especially among geriatric population [9]. Approximately $10 \%$ of the geriatric population suffers from severe anxiety and depressive symptoms [10]. Indeed, a study in six European countries has demonstrated that severe and stable joint pain is associated with anxiety and depressive symptoms among older adults with knee OA [11]. Moreover, depression is known to be a significant contributor to poorer health outcomes and to be the leading cause of disease burden worldwide [12]. According to the literature, functional outcomes in orthopedic procedure are also affected by depression, including lumbar spine surgeries [13], hip and knee arthroplasty [14, 15], shoulder surgery [16], and upper extremity pathologies $[17,18]$. However, to the best of our knowledge, there is a lack of reports on psychological factors associated with the self-reported outcomes in geriatric OA people undergoing IAHA

Given that the self-reported psychological symptoms of an individual affect his/her quality of life [19], we hypothesized that such symptoms (depression and anxiety in this study) have the same effects on knee functions, as reported by geriatric people who have undergone IAHA. In addition to psychological symptoms, pain is another important concern of people with OA [20]. Pain catastrophizing, broadly defined as a negative orientation toward actual or anticipated painful experiences [21], is reported to be significantly correlated with healthrelated quality of life in patients with knee OA [22]. Moreover, deterioration of pain for geriatric OA patients is also associated with the future loss in walking ability [23]. Therefore, we additionally hypothesized that pain also influences the knee functioning that is reported by geriatric people who have undergone IAHA.

This study used a longitudinal design to determine whether depression, anxiety, and pain that affect the patient-reported knee functions of $\mathrm{OA}$ patients who were older than 60 years and had undergone IAHA.

\section{Material and methods}

\section{Study design}

Patients who had been diagnosed with knee OA and attended orthopedic clinics for IAHA in one hospital in Taipei, Taiwan, were prospectively recruited from June 2016 to April 2018. Inclusion criteria of the eligible participants were (1) aged 60 years or above, (2) had suffered symptoms of knee OA, including pain and stiffness, for at least 3 months, and (3) had radiographic OA grades $2-3$ on the Kellgren and Lawrence grading scale [24]. Exclusion criteria were (1) had past or present trauma of, or surgery for cancer, malignant tumor or an infection in the target knee, (2) had a history of vasovagal shock and had used nonsteroidal antiinflammatory drugs (NSAIDs) within 2 days prior to HA injection, (3) had received corticosteroids by injection in the target knee in the preceding 6 months, or (4) had cognitive impairment, identified by the Short Portable Mental Status Questionnaire (SPMSQ) with a cut-off of five errors [25].

After written informed consent had been obtained, the basic demographic data (age, gender, marital status, educational level, and underlying comorbidities) of each patient were collected using a face-to-face interview. All participants completed the questionnaires mentioned below at baseline (before injection) and at three followups (at 2, 4, and 6 months). The questionnaires used the Geriatric Depression Scale (GDS), the State-Trait Anxiety Inventory (STAI), the Western Ontario and McMaster University Osteoarthritis Index (WOMAC), the International Knee Documentation Committee subjective knee evaluation form (IKDC), and the Visual Analogue Scale (VAS) of pain severity. Once they had completed the baseline survey, all participants underwent IAHA with a low-molecular-weight product of 2 $\mathrm{mL}$ sodium hyaluronate (SciVision Biotech Inc., Taiwan) with a molecular weight of 500-730 kDa once per week for 3 weeks. The protocol of IAHA is based on the manufacturing recommendations (http://www.scivision. com.tw/en/products_01_show.php?pc=4\&ps $=1$ ), which is a general treatment protocol in Wan Fang Hospital. At the follow-ups, all participants were permitted to choose simultaneous treatment with COX-II inhibitors (etoricoxib $60 \mathrm{mg}$ once daily) or physical therapy. Other treatments, including intraarticular injection with a steroid or platelet-rich plasma, were not permitted.

The entire protocol and instrumentation were approved by the ethical committee at Taipei Medical University, and the approval was registered as TMU-JIRB N201606003. All participants consented to the study and publication of data.

\section{Instruments}

\section{Measurement of psychological symptoms}

The GDS with 15 items is a validated tool to assess depression in older people by self-reporting [26, 27]. The state-anxiety subscale of the STAI with 20 items is a validated tool to assess subjective and transitory feelings of tension and nervousness by self-reporting [28]. A higher score on the GDS or the STAI indicates greater depression or anxiety, respectively. 


\section{Measurement of knee function}

The self-reported WOMAC with 24 items has three subscales (pain, stiffness, and physical function) and has promising psychometric advantages [29]. The selfreported IKDC with 18 items involves questions concerning symptoms, sports activities, and knee function. The IKDC also has promising psychometric benefits [30, 31]. A higher WOMAC or IKDC score indicates worse or better knee function, respectively.

\section{Measurement of pain}

The VAS is an instrument that is regularly used to measure pain intensity [32]. If both knees are injected, then the VAS score for the left side is recorded first and then that for the right side is recorded.

\section{Statistical analysis}

In addition to descriptive statistics for participant characteristics, numerous linear mixed effects models, which use restricted maximum likelihood estimation, were applied to understand the associations between patientreported outcomes (pain, anxiety, and depression) and knee functions (measured using IKDC and WOMAC). All of these models control for the time effects of the hyaluronic acid, gender, marital status, comorbidities, and age. Moreover, independent $t$ tests were applied to examine the outcome differences between patients who were grade 2 on the Kellgren and Lawrence grading scale and those who were grade 3. IBM SPSS 23.0 (IBM corp. Armonk, NY, USA) was used to conduct all of the analyses.

\section{Results}

After screening 149 participants, 47 were excluded and the retained 102 participants all agreed to participate in this study (Fig. 1). Among the 102 participants, 74 $(72.5 \%)$ were female, 80 received bilateral knee IAHA, and 22 undergone unilateral knee IAHA. Among the 182 knees within 102 patients, $20.3 \%$ of knees $(n=37)$ were defined as radiographic OA grade 3 on the Kellgren and Lawrence grading scale, whereas the other 145 knees were defined as radiographic OA grade 2 . The mean age of the participants was $70.91(\mathrm{SD}=7.19)$ years, and slightly more than one-quarter of them were males $(n=28)$. Almost all participants were married $(n$ $=73)$, and nearly $30 \%$ of them $(n=30)$ had a bachelor's or postgraduate degree. The most common comorbidity for the participants was hypertension $(n=63)$, which was followed by heart disease $(n=20)$, diabetes mellitus $(n=19)$, depression $(n=4)$, and cancer $(n=3)$. Table 1

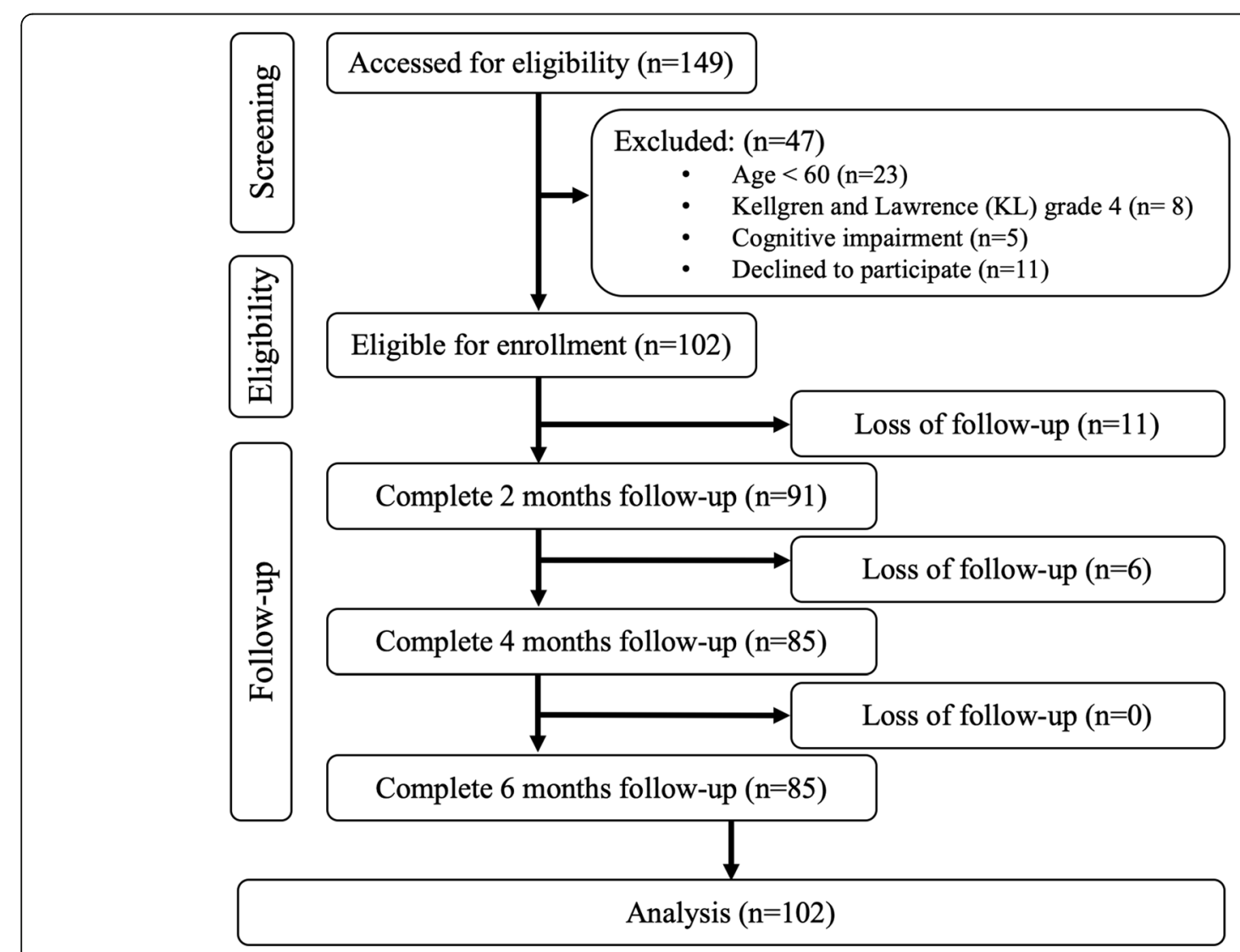

Fig. 1 Patient eligibility chart 
Table 1 Descriptive statistics in patient-reported outcomes and knee functions across time $(N=102)$

\begin{tabular}{|c|c|c|c|c|}
\hline & \multicolumn{4}{|l|}{$M(\mathrm{SD})$} \\
\hline & Baseline & 2 months & 4 months & 6 months \\
\hline$\overline{\text { STAl }^{a}}$ & $20.22(9.67)$ & $19.14(9.34)$ & $19.47(9.64)$ & $19.07(8.19)$ \\
\hline GDS & $2.75(2.56)$ & $2.99(2.57)$ & $2.96(2.37)$ & $2.87(2.27)$ \\
\hline VAS_L & $4.46(2.42)$ & $3.02(1.94)$ & $3.81(1.99)$ & $4.23(2.26)$ \\
\hline VAS_R & $4.23(2.01)$ & $3.04(1.99)$ & $4.04(2.04)$ & $4.42(2.12)$ \\
\hline IKDC & $52.94(13.23)$ & $56.16(12.28)$ & $54.26(12.17)$ & $52.74(12.12)$ \\
\hline WOMAC pain & $4.53(3.99)$ & $3.51(3.28)$ & $4.28(3.37)$ & $4.58(3.64)$ \\
\hline WOMAC stiffness & $1.58(1.70)$ & $1.21(1.45)$ & $1.40(1.47)$ & $1.58(1.51)$ \\
\hline WOMAC physical function & $14.75(13.84)$ & $11.78(10.24)$ & $12.98(10.24)$ & $13.95(10.75)$ \\
\hline WOMAC total score & $20.85(17.92)$ & $16.52(13.46)$ & $18.67(13.68)$ & $20.09(14.55)$ \\
\hline
\end{tabular}

VAS_L, Visual Analogue Scale on left knee pain; VAS_R, Visual Analogue Scale on right knee pain; GDS, Geriatric Depression Scale; IKDC, The International Knee Documentation Committee Questionnaire; WOMAC, Western Ontario and McMaster University Osteoarthritis Index

${ }^{a}$ State and trait anxiety inventory; only the state scale was used

provides the patient-reported outcomes and knee functions at four time points (baseline, and at 2-month, 4month, and 6-month follow-ups).

When confounders were controlled for, age was significantly associated with IKDC score (coefficients [SEs] $=-0.317[0.053] ; p<0.01$ ), the WOMAC physical function subscale score (coefficients [SEs] $=0.393$ [0.137]; $p$ $<0.01$ ), and the WOMAC physical function subscale score (coefficients [SEs] $=0.396$ [0.174]; $p<0.05$ ). Pain was significantly associated with the IKDC score (coefficients [SEs] $=-1.419$ and -1.337 [0.280 and 0.313]; $p<$ 0.001 , for left and right knee pain, respectively), the WOMAC pain subscale score (coefficients [SEs] $=0.280$ and 0.362 [0.107 and 0.118]; $p<0.01$, for left and right knee pain, respectively), the WOMAC stiffness subscale score (coefficient $[\mathrm{SE}]=0.151[0.053] ; p<0.01$, for left right knee pain), the WOMAC physical function subscale score (coefficient $[\mathrm{SE}]=1.158[0.353] ; p<0.01$, for right knee pain), and the WOMAC total score (coefficients [SEs] $=0.977$ and 1.515 [0.421 and 0.470] for left and right knee pain; $p<0.05$ and $<0.01$, respectively). Anxiety was significantly associated with the WOMAC pain subscale score (coefficient $[\mathrm{SE}]=0.056[0.028] ; p<$ $0.05)$. Depression was significantly correlated with the IKDC score (coefficient [SE] $=-1.064$ [0.259]; $p<$ 0.001 ), the WOMAC physical function subscale score (coefficient $[\mathrm{SE}]=1.348$ [0.292]; $p<0.001$ ), and the WOMAC total score (coefficient $[\mathrm{SE}]=1.595$ [0.389]; $p$ $<0.001$ ) (Table 2).

As for the outcome comparisons between patients with Kellgren and Lawrence grades 2 and 3, patients with radiographic OA grade 3 were significantly associated with higher baseline GDS $(p=0.01)$ and lower baseline IKDC score $(p=0.01)$ than patients with radiographic OA grade 2 (Table 3). Additionally, during 6month longitudinal follow-up, patients with radiographic OA grade 3 were also significantly associated with higher
STAI, higher GDS, higher WOMAC score, and lower IKDC score than patients with radiographic OA grade 2 (Table 3).

\section{Discussion}

This study demonstrates that knee pain and depression were the factors that most strongly and negatively affect the patient-reported knee functional outcomes, including IKDC and WOMAC scores, of elderly patients with knee OA who had undergone IAHA. Moreover, age is founded to be associated with poor patient-reported knee functional outcomes after IAHA. Additionally, patients with higher radiographic Kellgren and Lawrence grading (grade 3 ) presented with significantly more depression and poor knee functional outcomes than patients with lower radiographic OA grading (grade 2) at baseline and throughout 6-month longitudinal follow-up after IAHA.

Although IAHA is a commonly used intraarticular therapy for knee OA, controversy exists owing to the inconsistent results and conclusions of IAHA in the literature $[33,34]$. Unanimity has not yet been reached on the usefulness of IAHA for treating knee OA [35]. A recently published network meta-analysis revealed that IAHA is an effective short-term treatment option for pain due to knee OA and even more efficacious than NSAIDs, intraarticular corticosteroid, or placebo injection [36]. However, the efficacy of IAHA among geriatric OA patients varies because of the inconsistent characteristics of this population. High prevalence of anxiety and depression has been reported among geriatric OA patients [9], but no studies listed anxiety and depression as independent psychological factors affecting the outcome after IAHA. In our study, although no effect of anxiety on self-reported knee functions was observed, the effects of depression were found to be one of independent factors affecting functional outcomes after IAHA. Although 
Table 2 Effects of pain, anxiety, and depression on knee function

\begin{tabular}{|c|c|c|c|c|c|c|c|c|c|c|}
\hline \multirow[b]{3}{*}{ VAS_L } & \multicolumn{10}{|l|}{$B(\mathrm{SE})$} \\
\hline & \multicolumn{2}{|l|}{ IKDC } & \multicolumn{2}{|c|}{ WOMAC_P } & \multicolumn{2}{|c|}{ WOMAC_S } & \multicolumn{2}{|c|}{ WOMAC_F } & \multicolumn{2}{|c|}{ WOMAC_T } \\
\hline & -1.419 & $(0.280)^{* * *}$ & 0.280 & $(0.107)^{* *}$ & 0.151 & $(0.053)^{* *}$ & 0.516 & $(0.316)$ & 0.977 & $(0.421)^{*}$ \\
\hline VAS_R & -1.337 & $(0.313)^{* * *}$ & 0.362 & $(0.118)^{* *}$ & 0.055 & $(0.059)$ & 1.158 & $(0.353)^{* *}$ & 1.515 & $(0.470)^{* *}$ \\
\hline STAl ${ }^{a}$ & -0.077 & $(0.072)$ & 0.056 & $(0.028)^{*}$ & 0.003 & $(0.014)$ & 0.093 & $(0.081)$ & 0.148 & $(0.108)$ \\
\hline Geriatric depression scale & -1.064 & $(0.259)^{* * *}$ & 0.185 & $(0.098)^{\#}$ & 0.054 & $(0.049)$ & 1.348 & $(0.292)^{* * *}$ & 1.595 & $(0.389)^{* * *}$ \\
\hline 2 months (Ref: baseline) & -0.678 & $(0.867)$ & -0.262 & $(0.347)$ & -0.215 & $(0.175)$ & -1.250 & $(0.971)$ & -1.766 & $(1.306)$ \\
\hline 4 months (Ref: baseline) & -0.314 & $(1.046)$ & -0.007 & $(0.405)$ & -0.129 & $(0.202)$ & -1.015 & $(1.178)$ & -1.171 & $(1.573)$ \\
\hline 6 months (Ref: baseline) & -0.882 & $(1.156)$ & 0.042 & $(0.433)$ & 0.040 & $(0.215)$ & -0.740 & $(1.311)$ & -0.692 & $(1.736)$ \\
\hline Gender (Ref: female) & 3.878 & $(1.783)$ & 0.631 & $(0.567)$ & -0.397 & $(0.272)$ & -1.617 & $(2.101)$ & -1.417 & $(2.656)$ \\
\hline Married (Ref: single) & -14.214 & (5.380) & 1.513 & $(1.749)$ & 0.914 & $(0.844)$ & 2.258 & $(6.310)$ & 4.633 & (8.019) \\
\hline Widowed (Ref: single) & -17.211 & (5.679) & 2.404 & $(1.846)$ & 0.818 & $(0.891)$ & 3.957 & $(6.661)$ & 7.133 & $(8.465)$ \\
\hline Hypertension (Ref: yes) & 1.085 & (1.618) & -0.322 & $(0.514)$ & 0.188 & $(0.247)$ & -0.990 & $(1.907)$ & -1.184 & $(2.410)$ \\
\hline DM (Ref: yes) & 1.650 & $(1.954)$ & -0.125 & $(0.617)$ & 0.382 & $(0.296)$ & -3.531 & $(2.305)$ & -3.294 & $(2.908)$ \\
\hline Heart disease (Ref: yes) & 1.784 & $(2.007)$ & -1.326 & $(0.640)$ & -0.764 & (0.308) & -0.965 & $(2.363)$ & -3.044 & $(2.989)$ \\
\hline Depression (Ref: yes) & -8.059 & $(6.055)$ & 3.732 & $(1.961)$ & 0.445 & $(0.946)$ & 7.895 & $(7.107)$ & 12.162 & $(9.024)$ \\
\hline Cancer (Ref: yes) & 3.241 & (5.736) & -2.932 & (1.818) & 0.536 & $(0.873)$ & -5.338 & $(6.762)$ & -7.969 & (8.539) \\
\hline Age & -0.317 & $(0.117)^{* *}$ & -0.023 & $(0.037)$ & 0.025 & $(0.018)$ & 0.393 & $(0.137)^{* *}$ & 0.396 & $(0.174)^{*}$ \\
\hline
\end{tabular}

VAS L, Visual Analogue Scale on left knee pain; VAS $R$, Visual Analogue Scale on right knee pain; DM, diabetes mellitus; IKDC, The International Knee Documentation Committee Questionnaire; WOMAC, Western Ontario and McMaster University Osteoarthritis Index

${ }^{\#} p=0.061 ;{ }^{*} p<0.05 ;{ }^{* *} p<0.01 ;{ }^{* *} p<0.001$

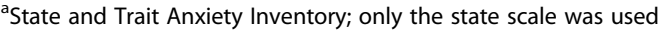

anxiety and depression are both psychological symptoms, depression is typically considered to be more severe than anxiety and is more like an affective disorder than a symptom [37]. Accordingly, depression, assessed using the GDS in this study, might have reflected the true mental health of the participants, whereas STAI (SAnxiety) might have reflected subjective and transitory feelings of tension or the nervousness experienced by participants at a given time. Therefore, healthcare providers should pay more attention to depression rather than to anxiety for geriatric people with knee OA who have undergone IAHA.

In addition, pain was also found to be one of independent factors affecting functional outcomes after IAHA in our study. Along with depression, pain is one of the evaluated items in both IKDC and WOMAC. Therefore, the strong association between the patientreported severity of pain based on VAS and the patientreported function outcomes based on IKDC and WOMAC in this study is unsurprising. In addition, pain has been reported to be an independent factor to predict the worsening of OA [38]. Pre-operative knee pain sensitization or pain catastrophizing was also reported to be associated with patients' satisfaction, quality of life, and functional improvement after total knee replacement $[21,39,40]$. Moreover, although only few studies discussed about the correction between pain and functional outcomes among geriatric OA patients receiving
IAHA, Bowman EN et al. revealed that pain score was strongly correlated with successful outcomes and appeared to be a reliable method to monitor treatment success after IAHA [41]. Our study demonstrated the similar finding that knee pain was one of the factors negatively affecting the patient-reported knee functional outcomes after IAHA for elderly OA patients.

Moreover, age was also found to be one of the important factors affecting the efficacy of IAHA [7]. Evidence has demonstrated that elderly OA patients may not benefit from IAHA as much as younger patients, who experience a longer period of functional improvement of the knee $[8,42]$. Hence, we postulated that the effects of IAHA are different between young-old OA patients (aged between 60 and 69 years) and old-old OA patients (aged 70 years or older). It is believed that elderly patients are vulnerable to worsen degeneration of cartilage as well as longer symptoms of knee OA so as to reflect the poor repose to IAHA. Our study also echoes the fact that age may negatively affect the patient-reported knee functional outcomes after IAHA. Additionally, more severe structural damage of knee was also an independent predictor for poor response to IAHA [8]. Indeed, our study demonstrated poor baseline and longitudinal selfreported functional outcome before and after IAHA in geriatric patients with higher radiographic OA grading. Also, these patients with more severe radiographic OA suffered more severe depressive status throughout the 


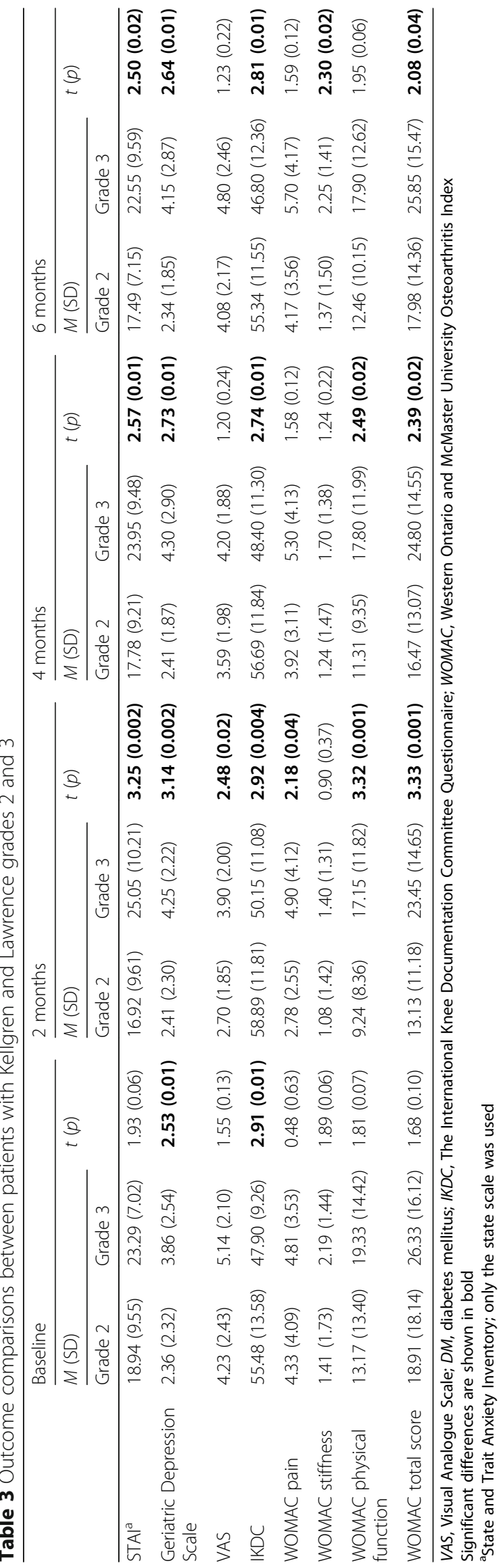


treatment duration with IAHA. Additional attention should therefore be paid to the potential influence of depression before IAHA especially among these geriatric OA patents with higher radiographic OA grading.

Our study has some limitations. First, the representativeness of our sample is somewhat questionable as we only recruited 102 elderly participants from the same institution, who might not represent the geriatric population with knee OA throughout Taiwan. Second, we did not exclude patients with other musculoskeletal disorders, except for knee OA. These musculoskeletal disorders might also contribute to chronic and subsequently affect the status of depression and anxiety among the geriatric population. Third, our causal relationship was not strong although a longitudinal design was adopted, and some degree of a causal relationship between psychological symptoms and knee functions was identified. Future studies using randomized controlled trials are warranted to corroborate our findings. Following the previous limitation, a longer period than our study follow-up is recommended because we believe that a 1year follow-up could have been observed for more significant results. However, even with these limitations, this study is the first to show that psychological symptoms may contribute to poor knee function that is reported by geriatric patients with knee OA who are undergoing IAHA.

\section{Conclusion}

Depression rather than anxiety was the most important psychological symptom that negatively affected the patient-reported knee functional outcomes of geriatric OA patients who had undergone IAHA. Further attention should be paid to the depressive status of geriatric people in cases of unsatisfactory outcomes of IAHA for knee OA that are reported by elderly patients.

\section{Abbreviations}

GDS: Geriatric Depression Scale; IAHA: Intraarticular hyaluronic acid injection; IKDC: International Knee Documentation Committee subjective knee evaluation form; NSAIDs: Nonsteroidal anti-inflammatory drugs; OA: Osteoarthritis; SPMSQ: Short Portable Mental Status Questionnaire; STAI: State-Trait Anxiety Inventory; VAS: Visual Analogue Scale; WOMAC: Western Ontario and McMaster University Osteoarthritis Index

\section{Acknowledgements}

We deeply appreciate all the participants.

\section{Authors' contributions}

Y-PC and C-YL drafted the paper. C-YL performed the statistical analysis. Y$P C, Y-Y H, Y W$, and Y-JK contributed to the interpretation of results. Y-PC, Y$\mathrm{YH}$, and $\mathrm{C}-\mathrm{YL}$ conceptualized the research design. All authors critically reviewed the manuscript during the writing process, and all authors approved the final version of the manuscript.

\section{Funding}

This research receives no funding.

\section{Availability of data and materials}

The datasets used and/or analyzed during the current study are available from the corresponding author on reasonable request.

Ethics approval and consent to participate

The present study protocol has been approved by the ethical committee at Taipei Medical University, and the approval was registered as TMU-JIRB N201606003.

\section{Consent for publication}

Not applicable.

\section{Competing interests}

The authors declare that they have no competing interests.

\section{Author details}

'Department of Orthopedic Surgery, Wan Fang Hospital, School of Medicine, College of Medicine, Taipei Medical University, Taipei, Taiwan. ${ }^{2}$ Graduate Institute of Nursing- Linkou campus, Chang Gung University of Science and Technology, Taoyuan, Taiwan. ${ }^{3}$ Department of Rehabilitation Sciences,

Faculty of Health and Social Sciences, The Hong Kong Polytechnic University, 11 Yuk Choi Rd, Hung Hom, Hong Kong.

Received: 16 January 2019 Accepted: 15 October 2019

Published online: 27 November 2019

\section{References}

1. Lawrence RC, Felson DT, Helmick CG, Arnold LM, Choi H, Deyo RA, et al. Estimates of the prevalence of arthritis and other rheumatic conditions in the United States. Part II. Arthritis Rheumatism. 2008:58:26-35.

2. Helmick CG, Felson DT, Lawrence RC, Gabriel S, Hirsch R, Kwoh CK, et al. Estimates of the prevalence of arthritis and other rheumatic conditions in the United States. Part I. Arthritis Rheum. 2008;58:15-25.

3. Zhang Y, Jordan JM. Epidemiology of osteoarthritis. Clin Geriatr Med. 2010; 26:355-69.

4. Richmond J, Hunter D, Irrgang J, Jones MH, Snyder-Mackler L, Van Durme D, et al. American Academy of Orthopaedic Surgeons clinical practice guideline on the treatment of osteoarthritis $(\mathrm{OA})$ of the knee. J Bone Joint Surg Am. 2010;92:990-3.

5. Johansen M, Bahrt H, Altman RD, Bartels EM, Juhl CB, Bliddal H, et al. Exploring reasons for the observed inconsistent trial reports on intraarticular injections with hyaluronic acid in the treatment of osteoarthritis: meta-regression analyses of randomized trials. Semin Arthritis Rheum. 2016; 46:34-48.

6. Day R, Brooks P, Conaghan PG, Petersen M, Multicenter Trial G. A double blind, randomized, multicenter, parallel group study of the effectiveness and tolerance of intraarticular hyaluronan in osteoarthritis of the knee. Rheumatol. 2004;31:775-82.

7. Newberry SJ, Fitzgerald JD, Maglione MA, O'Hanlon CE, Booth M, Motala A, et al. AHRQ technology assessments. Systematic review for effectiveness of hyaluronic acid in the treatment of severe degenerative joint disease (DJD) of the Knee. AHRQ technology assessments. Rockville: Agency for Healthcare Research and Quality (US); 2015.

8. Pelletier JP, Raynauld JP, Abram F, Dorais M, Delorme P, Martel-Pelletier J. Exploring determinants predicting response to intra-articular hyaluronic acid treatment in symptomatic knee osteoarthritis: 9-year follow-up data from the osteoarthritis initiative. Arthritis Res Ther. 2018;20:40.

9. Sugai K, Takeda-Imai F, Michikawa T, Nakamura T, Takebayashi T, Nishiwaki Y. Association between knee pain, impaired function, and development of depressive symptoms. J Am Geriatr Soc. 2018;66:570-6.

10. Al-Butmeh S, Al-Khataib N. Mental health and quality of life of elderly people in the Bethlehem district: a cross-sectional study. Lancet (London, England). 2018;391(Suppl 2):S46.

11. de Koning EJ, Timmermans EJ, van Schoor NM, Stubbs B, van den Kommer TN, Dennison EM, et al. Within-person pain variability and mental health in older adults with osteoarthritis: an analysis across 6 European cohorts. J Pain. 2018;19:690-8.

12. Moussavi S, Chatterji S, Verdes E, Tandon A, Patel V, Ustun B. Depression, chronic diseases, and decrements in health: results from the World Health Surveys. Lancet (London, England). 2007;370:851-8. 
13. Skolasky RL, Riley LH 3rd, Maggard AM, Wegener ST. The relationship between pain and depressive symptoms after lumbar spine surgery. Pain. 2012;153:2092-6.

14. Vissers MM, Bussmann JB, Verhaar JA, Busschbach JJ, Bierma-Zeinstra SM Reijman M. Psychological factors affecting the outcome of total hip and knee arthroplasty: a systematic review. Semin Arthritis Rheum. 2012:41:576-88.

15. Brander V, Gondek S, Martin E, Stulberg SD. Pain and depression influence outcome 5 years after knee replacement surgery. Clin Orthop Relat Res. 2007:464:21-6.

16. Dekker AP, Salar O, Karuppiah SV, Bayley E, Kurian J. Anxiety and depression predict poor outcomes in arthroscopic subacromial decompression. J Shoulder Elbow Surg. 2016;25:873-80.

17. Yeoh JC, Pike JM, Slobogean GP, O'Brien PJ, Broekhuyse HM, Lefaivre KA Role of depression in outcomes of low-energy distal radius fractures in patients older than 55 years. J Orthop Trauma. 2016:30:228-33.

18. London DA, Stepan JG, Boyer MI, Calfee RP. The impact of depression and pain catastrophization on initial presentation and treatment outcomes for atraumatic hand conditions. J Bone Joint Surg Am. 2014;96:806-14.

19. Lu IC, Yen Jean MC, Lei SM, Cheng HH, Wang JD. BSRS-5 (5-item Brief Symptom Rating Scale) scores affect every aspect of quality of life measured by WHOQOL-BREF in healthy workers. Qual Life Res. 2011:20:1469-75.

20. Riddle DL, Stratford PW. Knee pain during daily tasks, knee osteoarthritis severity, and widespread pain. Phys Ther. 2014;94:490-8.

21. Yakobov E, Stanish W, Tanzer M, Dunbar M, Richardson G, Sullivan MJL. The prognostic value of pain catastrophizing in health-related quality of life judgments after Total knee arthroplasty. Health Qual Life Outcomes. 2018; 16:126.

22. Somers TJ, Keefe FJ, Carson JW, Pells JJ, Lacaille L. Pain catastrophizing in borderline morbidly obese and morbidly obese individuals with osteoarthritic knee pain. Pain Res Manag. 2008;13:401-6.

23. Yamaguchi N, Takahashi T, Ueno T, Hiyama S, Ogawa M, Matsumura T, et al. Pain deterioration within 1 year predicts future decline of walking ability: a 7-year prospective observational study of elderly female patients with knee osteoarthritis living in a rural district. Geriatr Orthop Surg Rehabil. 2018;9: 2151459318799855.

24. Kellgren JH, Lawrence JS. Radiological assessment of osteo-arthrosis. Ann Rheum Dis. 1957;16:494-502.

25. Pfeiffer E. A short portable mental status questionnaire for the assessment of organic brain deficit in elderly patients. J Am Geriatr Soc. 1975;23:433-41.

26. Xie Z, Lv X, Hu Y, Ma W, Xie H, Lin K, et al. Development and validation of the geriatric depression inventory in Chinese culture. Int Psychogeriatr. 2015;27:1505-11.

27. Cruice M, Worrall L, Hickson L. Reporting on psychological well-being of older adults with chronic aphasia in the context of unaffected peers. Disabil Rehabil. 2011:33:219-28.

28. Shek DT. The Chinese version of the state-trait anxiety inventory: its relationship to different measures of psychological well-being. J Clin Psychol. 1993;49:349-58.

29. Quintana JM, Escobar A, Arostegui I, Bilbao A, Azkarate J, Goenaga Jl, et al. Health-related quality of life and appropriateness of knee or hip joint replacement. Arch Intern Med. 2006:166:220-6.

30. Xie F, Li S-C, Goeree R, Tarride J-E, O'Reilly D, Lo N-N, et al. Validation of Chinese Western Ontario and McMaster Universities Osteoarthritis Index (WOMAC) in patients scheduled for total knee replacement. Qual Life Res. 2008;17:595-601.

31. Irrgang JJ, Anderson AF, Boland AL, Harner CD, Kurosaka M, Neyret $P$, et al. Development and validation of the international knee documentation committee subjective knee form. Am J Sports Med. 2001;29:600-13.

32. Flandry F, Hunt JP, Terry GC, Hughston JC. Analysis of subjective knee complaints using visual analog scales. Am J Sports Med. 1991;19:112-8.

33. Rutjes AW, Juni P, da Costa BR, Trelle S, Nuesch E, Reichenbach S. Viscosupplementation for osteoarthritis of the knee: a systematic review and meta-analysis. Ann Intern Med. 2012;157:180-91.

34. Bellamy N, Campbell J, Robinson V, Gee T, Bourne R, Wells G. Viscosupplementation for the treatment of osteoarthritis of the knee. Cochrane Database Syst Rev. 2006:CD005321.

35. Altman RD, Schemitsch E, Bedi A. Assessment of clinical practice guideline methodology for the treatment of knee osteoarthritis with intra-articular hyaluronic acid. Semin Arthritis Rheum. 2015;45:132-9.

36. Bannuru RR, Schmid CH, Kent DM, Vaysbrot EE, Wong JB, McAlindon TE. Comparative effectiveness of pharmacologic interventions for knee osteoarthritis: a systematic review and network meta-analysis. Ann Intern Med. 2015;162:46-54

37. Chang CC, Wu TH, Chen CY, Lin CY. Comparing self-stigma between people with different mental disorders in Taiwan. J Nerv Ment Dis. 2016;204:547-53.

38. Eyles JP, Mills K, Lucas BR, Williams MJ, Makovey J, Teoh L, et al. Can we predict those with osteoarthritis who will worsen following a chronic disease management program? Arthritis Care Res (Hoboken). 2016;68:1268-77.

39. Kornilov N, Lindberg MF, Gay C, Saraev A, Kuliaba T, Rosseland LA, et al. Higher physical activity and lower pain levels before surgery predict nonimprovement of knee pain 1 year after TKA. Knee Surg Sports Traumatol Arthrosc. 2018:26:1698-708.

40. Arendt-Nielsen L, Simonsen O, Laursen MB, Roos EM, Rathleff MS, Rasmussen $\mathrm{S}$, et al. Pain and sensitization after total knee replacement or nonsurgical treatment in patients with knee osteoarthritis: identifying potential predictors of outcome at 12 months. Eur J Pain. 2018;22:1088-102.

41. Bowman EN, Hallock JD, Throckmorton TW, Azar FM. Hyaluronic acid injections for osteoarthritis of the knee: predictors of successful treatment. Int Orthop. 2018;42:733-40.

42. Ucar D, Diracoglu D, Suleyman T, Capan N. Intra-articular hyaluronic acid as treatment in elderly and middle-aged patients with knee osteoarthritis. Open Rheumatol J. 2013;7:38-41.

\section{Publisher's Note}

Springer Nature remains neutral with regard to jurisdictional claims in published maps and institutional affiliations.
Ready to submit your research? Choose BMC and benefit from:

- fast, convenient online submission

- thorough peer review by experienced researchers in your field

- rapid publication on acceptance

- support for research data, including large and complex data types

- gold Open Access which fosters wider collaboration and increased citations

- maximum visibility for your research: over $100 \mathrm{M}$ website views per year

At $\mathrm{BMC}$, research is always in progress.

Learn more biomedcentral.com/submissions 